\title{
Influence of a prolonged period of low-dosage $x$-rays on the optic and ultrastructural appearances of cataract of the human lens
}

\author{
B. P. HAYES AND R. F. FISHER \\ From the Department of Visual Science, Institute of Ophthalmology, London
}

SUMMARY Human lenses extracted for cataract 26 years after long-term exposure to an imperfectly shielded radium source were examined by slit-lamp photography, thin-section light microscopy, and electron microscopy. Anterior epithelial cells were fibroblast-like, and germinal epithelium and vacuolated cortical fibres had accumulated at the equator. A zone of light scatter at the anterior pole corresponded to an area of breakdown of cortical lens fibres, where unusual feathery fibres were orientated perpendicular to the lens surface. Two zones of light scatter separated by a $250-\mu \mathrm{m}$ clear interval were seen in the posterior cortex. The zone at the posterior pole corresponded to an area of fibre liquefaction and large rounded membrane whorls, while the deeper zone comprised small flattened membrane whorls. The characteristic plaques of swollen abnormal cells described in previous histological studies of $x$-ray cataract were not present. This and other differences probably reflect the extremely long time course and repeated subliminal doses to which the patient was exposed.

Previous histological studies of both human and experimental $x$-radiation cataracts have mainly dealt with short-term changes developing after high radiation doses (for references see Duke-Elder, 1972; Hanna, 1975; Palva and Palkama, 1978). These studies indicate that $x$-rays act primarily on the germinal epithelial cells at the lens equator, and the cells divide to form defective lens fibres, which accumulate in plaques near the anterior and posterior poles. The breakdown of these defective fibres gives characteristic opacities in an anterior subcapsular zone and disc-shaped opacities in the posterior cortex (Hogan and Zimmerman, 1962).

In this study slit-lamp photography, and light and electron microscopy are used to investigate cataractous changes in human lenses which appeared many years after prolonged exposure to repeated low doses of $x$-radiation over a period of 11 years. The structure of the lenses is compared with that found in previous studies of $x$-ray cataract, in normal lenses (Hogan et al., 1971), and senile cataract.

\section{Materials and methods}

The lenses were obtained after routine cataract extraction from a patient whose visual acuity had been reduced to $6 / 24$ in each eye. Before cataract

Address for reprints: Dr B. P. Hayes, Institute of Ophthalmology, Judd Street, London WC1H 9QS extraction the patient had been subjected to an imperfectly shielded radium source $(120 \mathrm{mg})$ for a few minutes 3 times a week for a period of 11 years (1935-50). From 1950 there had been no exposure to radiation until removal of his cataract 26 years later.

Whole lenses were fixed by immersion in 4 changes of $3 \%$ glutaraldehyde in $0.1 \mathrm{M}$ Sorenson's phosphate buffer pH 7.3 containing $0.1 \% \mathrm{CaCl}_{2}$ for 4 hours at $4^{\circ} \mathrm{C}$. They were washed 10 times with the same buffer with $9 \%$ sucrose added and postfixed in 4 changes of $1 \% \mathrm{OsO}_{4}$ in White's solution (Wakely, 1974) overnight at $4^{\circ} \mathrm{C}$. The lenses were bisected through the lens axis, block stained with buffered uranyl acetate (Brightman and Reese, 1969), dehydrated, and then embedded in Araldite. Light and electron microscope sections were cut approximately perpendicular to the surface of the lens in the regions of the anterior pole, equator, and posterior pole. Light microscope sections were stained with $1 \%$ toluidine blue/borax and electron microscope sections with ethanolic uranyl acetate and alkaline lead citrate (Reynolds, 1963).

\section{Results}

SLIT-LAMP APPEARANCES

Fig. 1. View of anterior cortex. There is a dense area of scatter just below the capsule in the region of the anterior pole of the lens, and also isolated areas 

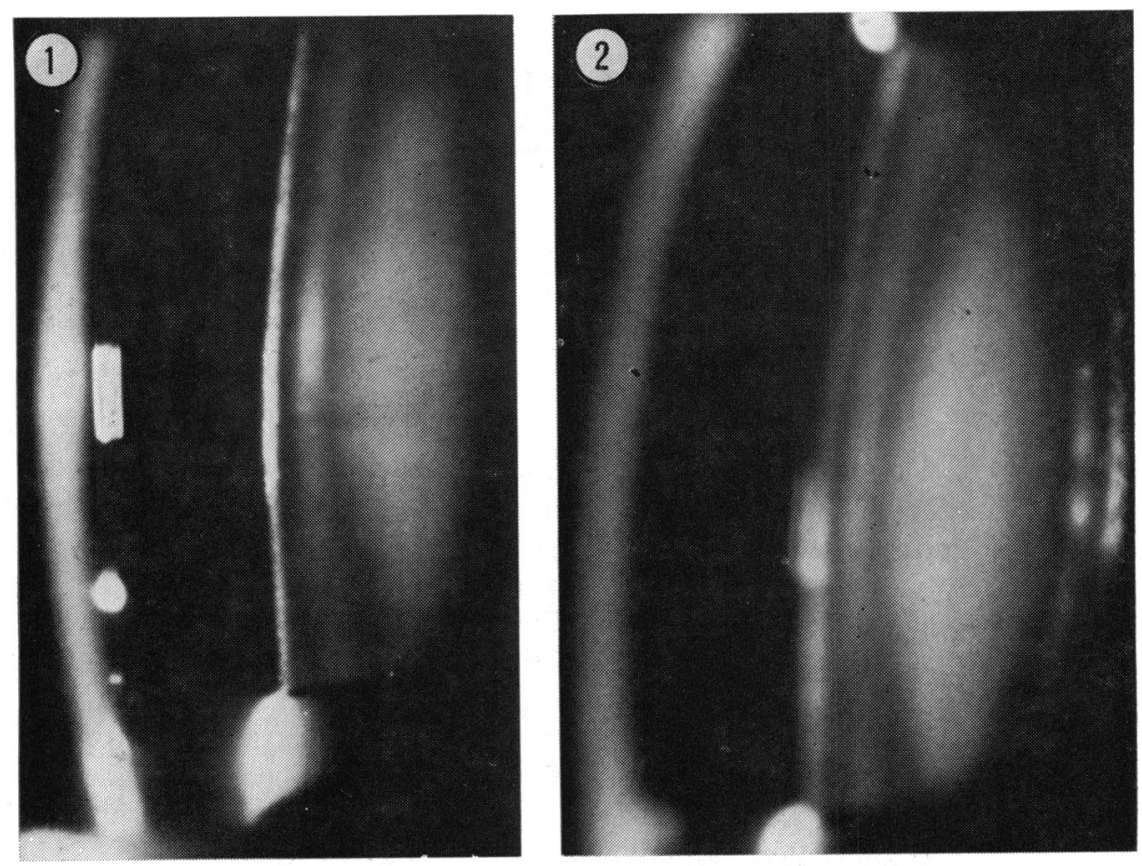

Fig. 1 Slit-lamp view of anterior portion of the lens showing subcapsular opacification

Fig. 2 Slit-lamp view of posterior portion of the lens showing two areas of scatter separated by a clear interval

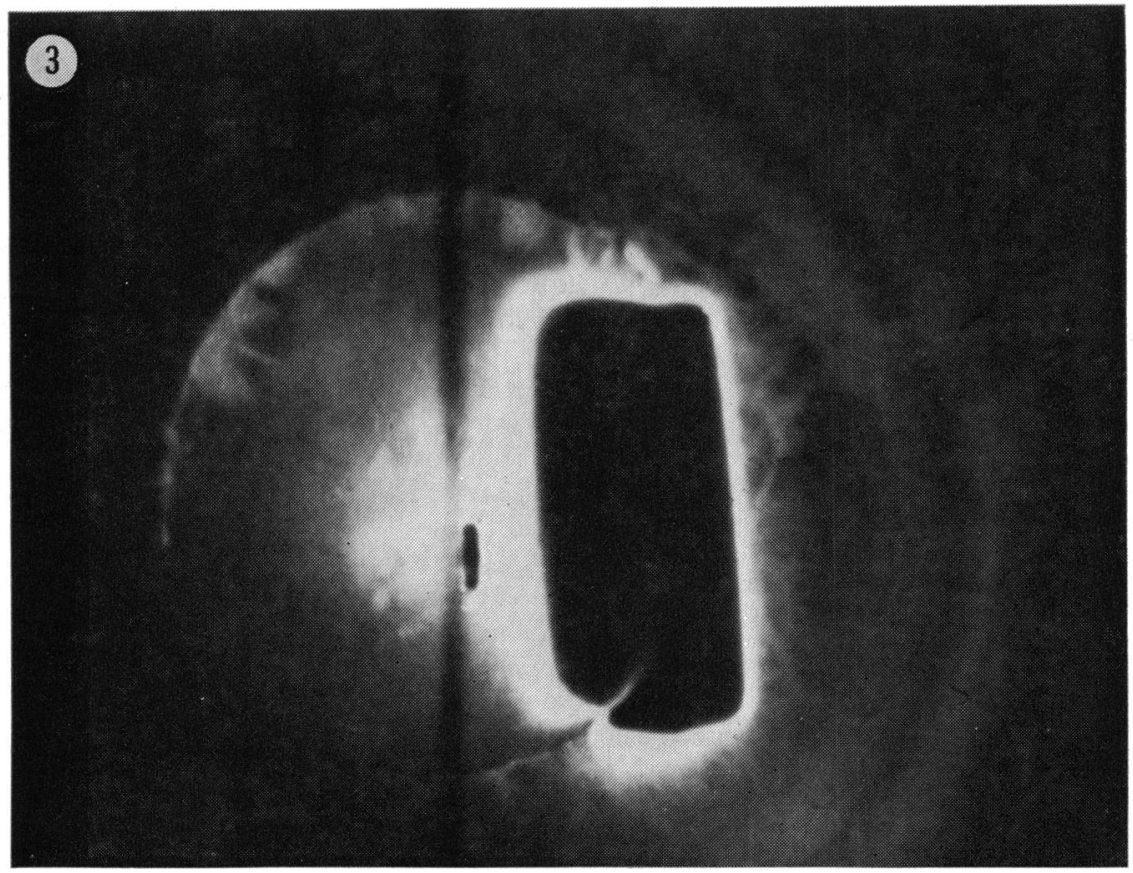

Fig. 3 Slit-lamp view of posterior portion of the lens by reflected light showing some isolated areas of scatter at the periphery of the opacity

which are again subcapsular out as far as the edge of the iris. The electron microscopy of these areas is shown in Figs. 4-10 and corresponds to feather fibres and the large vacuolated cells.

Fig. 2. View of posterior cortex. Two yellowish areas of scatter are seen in the posterior cortex separated by a clear interval. At the posterior pole of the lens the scatter area corresponds to Fig. 11. The other area between the posterior pole and nucleus corresponds to Fig. 14. Neither area has a 
Fig. $41 \mu \mathrm{m}$ light microscope section of the anterior capsule, epithelium, and lens cortex. The asterisk marks a local thickening of the lens capsule from 15 to $28 \mu \mathrm{m}$. The spindle-shaped epithelial cells (e) are very darkly stained. Lens fibres show swelling, shrinkage, and dark granules. $\times 490$; scale line $10 \mu \mathrm{m}$

Fig. 5 Spindle-shaped anterior epithelial cell with rounded processes (arrows) extending into the cortex. The cell contains many densely stained mitochondria (m). $\times 8900$; scale line $1 \mu \mathrm{m}$
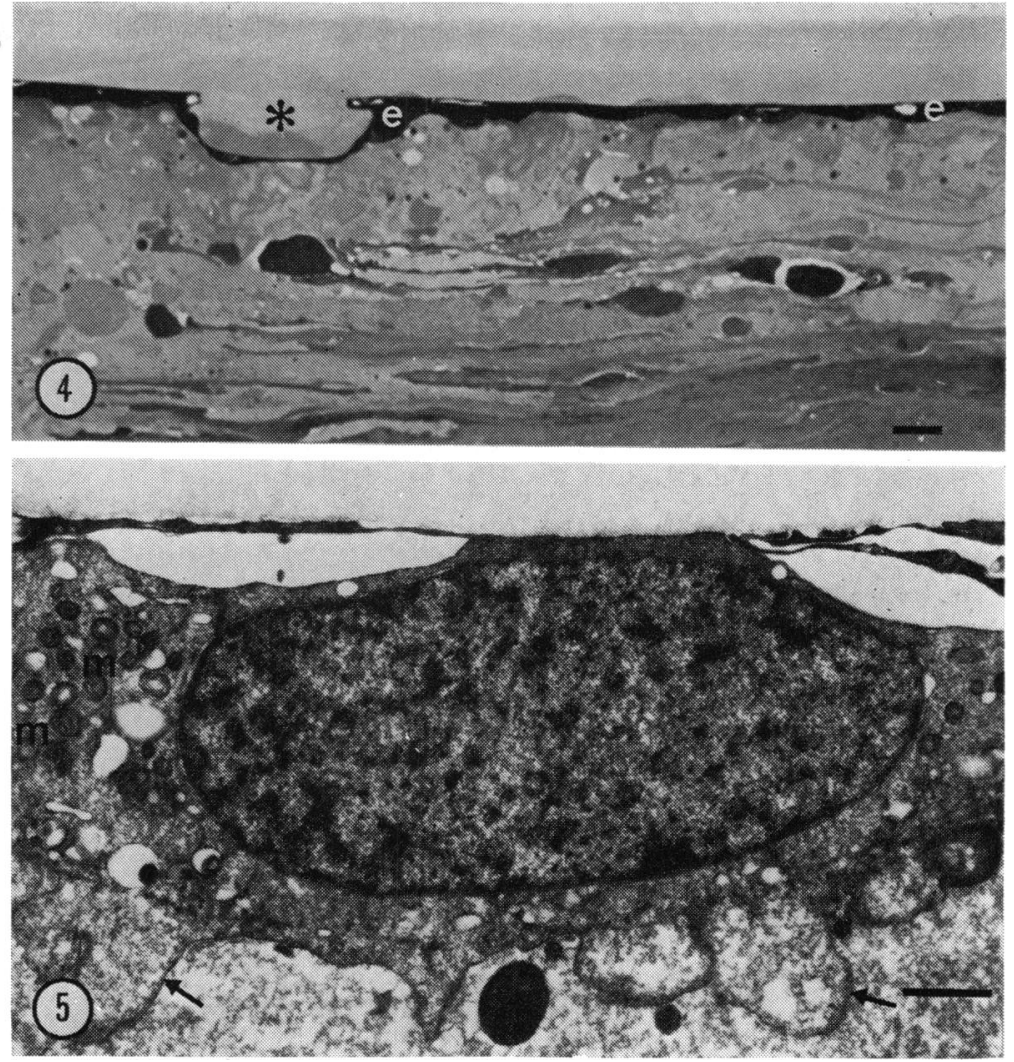

typical bivalve appearance which is seen in severe radiation cataract, for example, atomic bomb radiation. In addition at the periphery there are some irregular areas of scatter which do not correspond to cuneiform-like opacities which would be seen on retro-illumination. These areas produce scatter by reason of the irregularity of the lens fibres, which are overlaid by vacuolated areas (Fig. 10).

Fig. 3. View by retro-illumination. A circular area of increased scatter is shown at the posterior pole of the lens together with some irregular areas at the lens periphery. These appearances do not show the markedly increased scatter at its edge producing a ring-shaped bounded area characteristic of acute radiation cataract. However, the margins of the area are more clearly defined when compared with senile cupuliform cataract. (From the measurement of slit-lamp photographs the clear interval between these areas is about $250 \mu \mathrm{m}$ thick. This corresponds to a period of about 30 to 35 years for the fibres to be laid down [Fisher, 1970].)

LIGHT AND ELECTRON MICROSCOPY Anterior lens. The thin epithelium at the anterior pole (1 to $3 \mu \mathrm{m}$ thick) comprised darkly stained spindle-shaped cells up to $30 \mu \mathrm{m}$ long (Fig. 4). Occasional breaks were found in the epithelium, and many cells contained large vacuoles. The capsule at the anterior pole was usually about $17 \mu \mathrm{m}$ thick, but a small area was found where capsule thickness increased to $29 \mu \mathrm{m}$ (Fig. 4); the spindleshaped epithelial cells were here depressed into the lens cortex. Away from the anterior pole the epithelium was thicker $(5 \mu \mathrm{m})$ and the cells were less densely stained. These epithelial cells were unusual in having many dark mitochondria and a number of rounded projections into the lens cortex (Fig. 5).

An area of liquefaction of the superficial cortical lens fibres, $200 \mu \mathrm{m}$ deep, was observed at the anterior pole, containing feathery filaments orientated mainly perpendicular to the lens surface (Fig. 6). Electron microscopy showed that the matrix of this watery area and of the filaments resembled the fine granular cytoplasmic matrix of intact lens fibres (Fig. 7). The feathery filaments, a maximum of $20 \mu \mathrm{m}$ wide and $200 \mu \mathrm{m}$ long, were only partly contained by cell membrane, junctional membranes being particularly well preserved. Among the filaments dense granules 

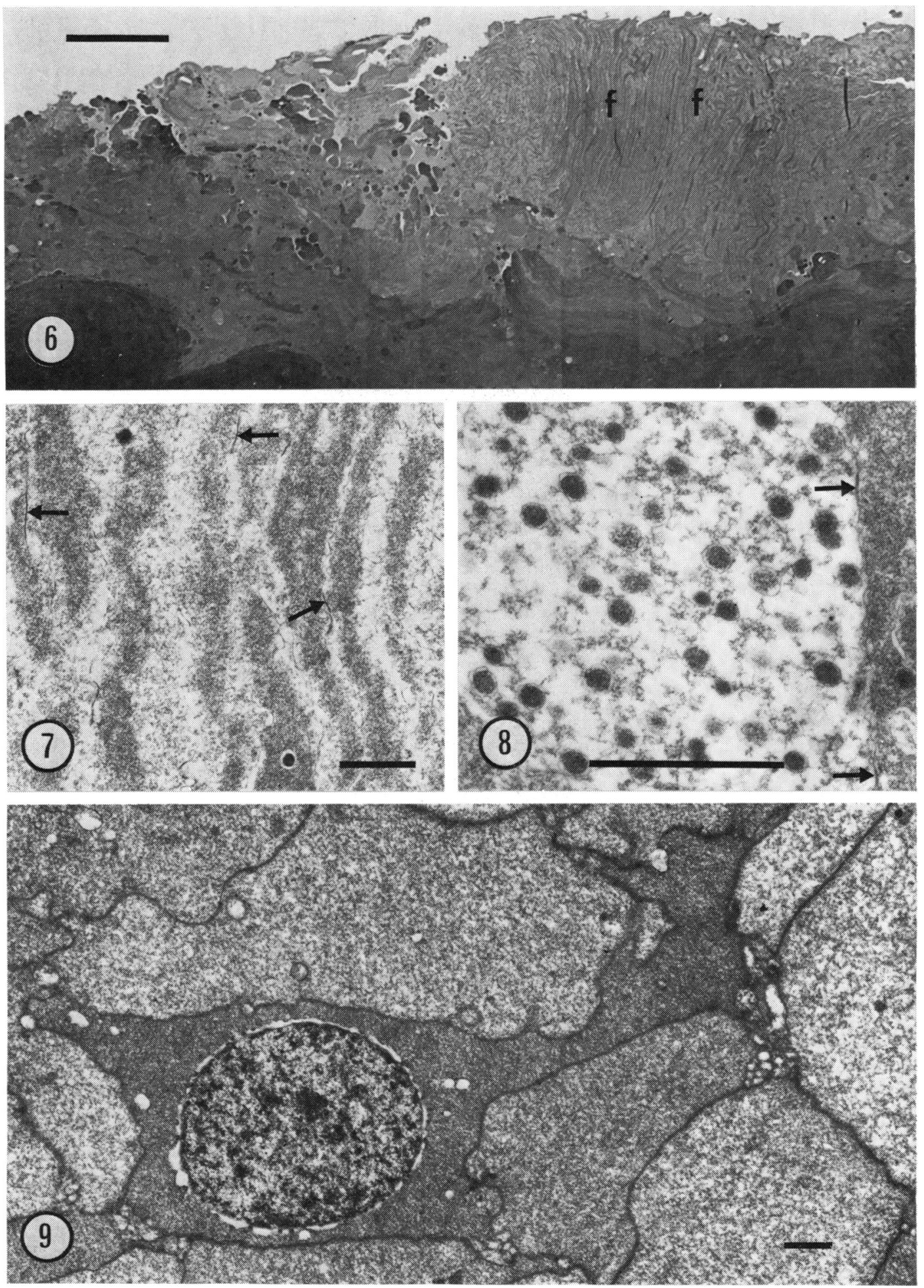
$(0.1$ to $0 \cdot 3 \mu \mathrm{m}$ diameter $)$ were found. At the edge of the filamentous area cortical lens fibres were swollen and dense membrane-bound granules, up to $1 \mu \mathrm{m}$ diameter, were common (Fig. 8). Swollen lens fibres reached $10 \mu \mathrm{m}$ diameter and were bounded by fragmentary cell membranes, occasionally fused to small membrane whorls (Fig. 6). Other superficial cortical fibres (to a depth of about $50 \mu \mathrm{m}$ ) had a more normal ultrastructural appearance although they varied in diameter (up to $8 \mu \mathrm{m}$ ) and were undulating in outline (Fig. 9). Nucleated fibres with amoeboid outlines were occasionally seen among them (Fig. 9). Deeper cortical fibres were dense and shrunken with wavy outlines (Figs. 4 and 6).

Equator. Towards the equator the epithelial cells gradually became less spindle shaped and more cuboidal in shape, the epithelium was about $10 \mu \mathrm{m}$ thick (normal thickness $15 \mu \mathrm{m}$ ), and the cells contained many vacuoles next to the capsule (Fig. 10). Between the epithelium and lens fibres was a darkly stained layer about $0.5 \mu \mathrm{m}$ deep. At the equator the germinal epithelium formed a plaque of cells with oval palely stained cell bodies about 3 cells $(22 \mu \mathrm{m})$ thick. Extending anteriorly beneath the epithelium and posteriorly beneath the capsule for about $200 \mu \mathrm{m}$ was a layer of unusually fine fibres with diameters of $1 \mu \mathrm{m}$ or less and containing many small vacuoles. Beneath these fibres the superficial cortex appeared to be normal to a depth of $90 \mu \mathrm{m}$ where fibre swelling was marked. Deeper in the cortex fibres were darkly stained, undulating, and fragmented as in the deep anterior cortex.

Fig. 6 Light micrograph of lens cortex near the anterior pole. Many swollen and shrunken lens fibres and fine granules can be seen on the left of the micrograph, and on the right feathery fibres $(f)$ are orientated perpendicular to the lens surface. The capsule was lifted away from the anterior lens during dissection. $\times 160$; scale line $100 \mu \mathrm{m}$

Fig. 7 Electron micrograph of the feathery fibres shown in Fig. 6. Fragments of cell membrane occur between them. Junctional membrane: arrows. $\times 11800$; scale line $1 \mu \mathrm{m}$

Fig. 8 Intracellular granules and junctional membranes (arrows) of the swollen lens fibres in the anterior cortex. The dense granules have a similar matrix to the lens fibres and most are approximately $0.1 \mathrm{\mu m}$ diameter. $\times 30000$; scale line $1 \mathrm{\mu m}$

Fig. 9 Nucleated cell in the anterior cortex has processes extending between the swollen misshapen lens fibres. $\times 7600$; scale line $1 \mathrm{\mu m}$
Posterior lens. The lens capsule at the posterior pole, which had artefactually lifted away from the cortex, was of normal thickness (approx. $3 \mu \mathrm{m}$ ). The posterior pole showed liquefaction of cortical lens fibres to a depth of $150 \mu \mathrm{m}$ in the right and $500 \mu \mathrm{m}$ in the left lens. The watery area contained little granular or filamentous debris when compared with the anterior pole (compare Figs. 6 and 11). Debris in the watery area was in the form of dense membrane-bound granules up to $5 \mu \mathrm{m}$ diameter and membrane fragments and whorls (Fig. 12). At the margin of the area of liquefaction the shrunken wavy cortical fibres had swollen rounded tips (Fig. 11), and similar rounded profiles with a matrix like that of cortical lens fibres were found in the watery area (Figs. 11 and 12). Subcapsular fibres at the posterior surface to the side of the watery area were disorganised to a depth of $40 \mu \mathrm{m}$, some being swollen while others were dense and appeared shrunken (Fig. 13). Large rounded membrane whorls, maximum diameter $2 \mu \mathrm{m}$, were found between the fibres, made up of stacks of fused membranes with a complex folded pattern (Fig. 13). In the right lens a further zone of membrane whorls was observed parallel to the posterior lens surface and localised beneath the watery area at 200 to $260 \mu \mathrm{m}$ depth. Here the membrane whorls were flattened parallel to the lens fibres and the fused membranes continuous with the lens fibre cell membranes (Fig. 14).

\section{Discussion}

When the fine structure of the cataractous lenses is compared to that of the normal human lens (Hogan et al., 1971; Kobayashi and Suzuki, 1975), the greatest changes are seen in the lens epithelium, anterior and posterior cortex. The lens capsule has a normal appearance and thickness except for a local thickening near the anterior pole, where the epithelium is depressed into the cortex. Local depressions of this type are thought to be the sites of elimination of lens fibre debris into the capsule and are common in senile cataract (Brini et al., 1963). In a histological study of ionising radiation cataracts by Cogan et al. (1952) thickening of the posterior capsule was, however, consistently observed.

Other common features of previous histological studies of $x$-radiation cataract are subcapsular plaques of epithelial cells, particularly at the posterior pole, where these are often swollen to form bladder or Wedl cells (Cogan and Donaldson, 1951; Cogan et al., 1952; Fujinaga, 1973; Hanna, 1975; Palva and Palkama, 1978). No plaques are found in this study, but rare nucleated lens fibres occur in the anterior cortex. The anterior epithelium is extremely thin 

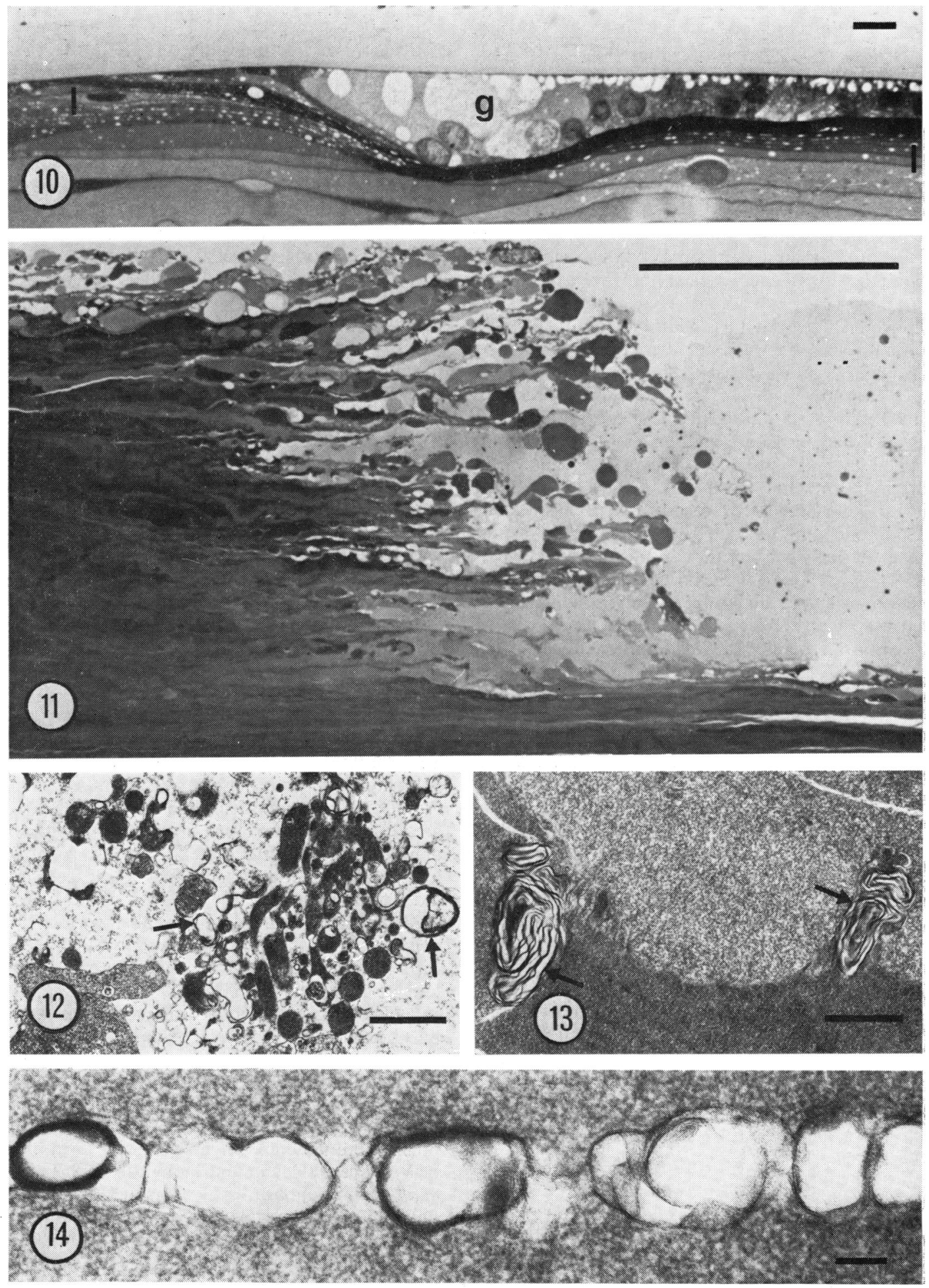
and discontinuous; the epithelial cells are spindle shaped in section and about $30 \mu \mathrm{m}$ diameter instead of their normal $20 \mu \mathrm{m}$ (Hogan et al., 1971). There is reduced interdigitation between the cells; they contain many darkly stained mitochondria and have projections into the cortex (see Palva and Palkama, 1978). This patchy, fibroblast-like anterior epithelium has previously been found after irradiation (Cogan et al., 1952; Hanna, 1975).

Low doses of radiation are thought to act on the germinal epithelial cells at the lens equator causing accelerated defective cell division (Hanna, 1975). Lens fibres formed from these cells fail to elongate, lose their cellular attachments, and become vacuolated. In this study an accumulation of epithelial cells and shrunken vacuolated cortical fibres occurs at the equator suggesting that the germinal epithelium has been damaged and is unable to form normal lens fibres. Other superficial equatorial cortical fibres are slightly swollen but otherwise normal (Hogan et al., 1971). Deeper cortical fibres are darkly stained, shrunken, and fragmented like normal nuclear fibres or the cortical fibres of senile cataracts (Kobayashi and Suzuki, 1975).

Zones of cortical liquefaction are found at the anterior and posterior poles consistent with the clinical appearance of late stage $x$-ray cataract, that is, disc-shaped polar cortical opacities (DukeElder, 1972). At the edges of the liquefied zones a

Fig. 10 Lens equator. Epithelial cells and superficial cortical lens fibres are vacuolated. Germinal epithelial cells $(g)$ have accumulated at the equator and a layer of thin vacuolated lens fibres (l) can be seen in the superficial cortex. $\times 650$; scale line $100 \mu \mathrm{m}$

Fig. 11 Posterior cortex. Shrunken wavy lens fibres on the left of the micrograph have swollen tips extending into the area of liquefied lens cortex on the right. Round granules are suspended in the liquefied area which is near the posterior pole. $\times 400$; scale line $100 \mu \mathrm{m}$

Fig. 12 Debris in the liquefied area of the posterior lens cortex consists of membrane-bound granules and membrane whorls (arrows). $\times 11800$; scale line $1 \mathrm{\mu m}$

Fig. 13 Posterior cortex bordering the liquefied area. Large rounded membrane whorls (arrows) are found between the distorted superficial cortical lens fibres just below the capsule. $\times 11800$; scale line $1 \mathrm{\mu m}$

Fig. 14 Flattened membrane whorl in the posterior cortex at a depth of about $250 \mu \mathrm{m}$ below the capsule. Up to 5 membranes are fused together to form the whorl. $\times 78000$; scale line $0.1 \mathrm{\mu m}$ variety of degenerative changes are seen in the cortical lens fibres-shrinkage of fibres and breakdown of their cell membranes, swelling of fibre tips, and the formation of small dense intracellular membrane-bound granules. The watery areas contain the products of this degeneration. For instance, the large round globules are probably the pinched-off swollen tips of cortical fibres and may correspond to the morgagnian globules found in more mature cortical cataracts (Hogan and Zimmerman, 1962). Membrane fragments and small dense granules are also found here.

The most striking feature of the anterior area of liquefaction is the feathery fibres orientated perpendicular to the lens surface, bounded by incomplete cell membranes. These shrunken realigned cortical fibres do not appear to have been described in previous histological studies of cataract. In the posterior cortex bordering the watery area damage to the cortical fibre cell membranes is apparent both as fragmentation and as large rounded membrane whorls of fused cell membranes. Somewhat larger whorls are found in mature posterior cortical cataract (Hayes, unpublished). The yellowish area of light scatter seen in the slit lamp at the posterior pole probably includes both the watery area and the surrounding region of cortical fibre membrane damage.

A deeper zone of light scatter between the pole and the nucleus corresponds closely in depth and lateral spread with the distribution of flattened membrane whorls. This may be the original zone of radiation damage to the posterior lens, subsequently buried in the lens cortex by the growth of more normal lens fibres in the 26 years after exposure to radiation (see Fisher, 1970). Fisher and Wakely (1976) showed experimentally that zones of light scatter in the lens can be produced by membrane whorls which develop several years after damage to the capsule, and Philipson (1973) has also suggested that membrane whorls cause a loss of transparency in supranuclear cuneiform cataract.

We are most grateful to Mr S. Miller for the clinical material examined in this study. We would also like to thank the Moorfields Research Grant for financial support. The electron microscope used for this study was donated by the Wellcome Foundation.

\section{References}

Brightman, M. W., and Reese, T. S. (1969). Junctions between intimately apposed cell membranes in the vertebrate brain. Journal of Cell Biology, 40, 648-677.

Brini, A., Porte, A., and Stoekel, M. E. (1963). Modifications ultrastructurals du crystallin dans certaines cataractes expérimentales et humaines. Bulletins et Mémoires de la Société Française d'Ophtalmologie, 76, 193-208.

Cogan, D. G., and Donaldson, D. D. (1951). Experimental radiation cataracts: cataracts in the rabbit following single 
$x$-ray exposure. Archives of Ophthalmology, 45, 508-522.

Cogan, D. G., Donaldson, D. D., and Reese, A. B. (1952). Clinical and pathological characteristics of radiation cataract. Archives of Ophthalmology, 47, 55-70.

Duke-Elder, S. (1972). Injuries, Pt. 2, Non-mechanical Injuries. In System of Ophthalmology, Vol. XIV, pp. 985999. Kimpton: London.

Fisher, R. F. (1970). Senile cataract: a comparative study between lens fibres stress and cuneiform opacity formation. Transactions of the Ophthalmological Societies of the United Kingdom, 90, 93-109.

Fisher, R. F., and Wakely, J. (1976). Changes in lens fibres after damage to the lens capsule. Transactions of the Ophthalmological Societies of the United Kingdom, 96, 278-284.

Fujinaga, Y. (1973). Atomic bomb radiation cataract. Acta Societatis Ophthalmologica Japonicae, 77, 305-309.

Hanna, C. (1975). Cataract of toxic etiology: A. Radiation cataract. In Cataract and Abnormalities of the Lens, pp. 217-224. Edited by J. G. Bellows. Grune \& Stratton: New York.
Hogan, M. J., Alvarado, J. A., and Weddell, J. E. (1971). Histology of the Human Eye, pp. 638-677. Saunders: Philadelphia.

Hogan, M. J., and Zimmerman, L. E. (1962). Ophthalmic Pathology, pp. 682-683. Saunders: Philadelphia.

Kobayashi, Y., and Suzuki, T. (1975). The aging lens: ultrastructural changes in cataract. In Cataract and Abnormalities of the Lens, pp. 313-343. Edited by J. G. Bellows. Grune \& Stratton: New York.

Palva, M., and Palkama, A. (1978). Ultrastructural lens changes in $x$-ray induced cataract of the rat. Acta Ophthalmologica, 56, 587-598.

Philipson, B. (1973). Changes in the lens related to the reduction in transparency. Experimental Eye Research, 16, 29-39.

Reynolds, E. S. (1963). The use of lead citrate at high pH as an electron opaque stain in electron microscopy. Journal of Cell Biology, 17, 208-212.

Wakely, J. (1974). Senile changes in the fine structure of the lens in the rudd (Scardinius eryophthalmicus). Experimental Eye Research, 18, 571-577. 